\title{
Ocorrência de parasitos zoonóticos em fezes de cães provenientes de uma entrequadra da asa norte de Brasília, DF
}

\author{
Aline Silva Moraes \\ Diogo Claudino² \\ Juliana Vieira Ferreira Name ${ }^{3}$ \\ Laiane da Silva Carneiro ${ }^{4}$ \\ Loiane Alves Vieira ${ }^{5}$ \\ Daniella de Sousa Mendes Moreira Alves ${ }^{6}$
}

\section{Resumo}

O conhecimento da frequência de parasitos em cães é importante devido ao caráter zoonótico e aos danos que causam aos hospedeiros. Foram analisadas 20 amostras de fezes de cães, destas, 7 foram colhidas em domicílio e 13 encontradas em locais públicos da Asa Norte-DF. Oito apresentaram ovos e cistos de parasitos de relevância clínica tais como os do gênero Toxocara sp., Giardia sp., ancilostomídeo. Este estudo foi importante para o esclarecimento da população local sobre o risco de contaminação por fezes de cães. A partir dele sugere-se que medidas de controle sejam intensificadas para garantir melhor qualidade de vida à população.

Palavras-chave: Fezes de cães. Técnicas de diagnóstico parasitológico. Frequência de parasitos.

${ }^{1}$ Estudante do curso de Biomedicina no UniCEUB. E-mail: alinesilvamoraes_df@yahoo. com.br.

${ }^{2}$ Estudante do curso de Biomedicina no UniCEUB. E-mail: diogo.claudino@gmail.com.

${ }^{3}$ Estudante do curso de Biomedicina no UniCEUB. E-mail: jvname@gmail.com.

${ }^{4}$ Estudante do curso de Biomedicina no UniCEUB. E-mail: laiane_bio@yahoo.com.br.

5 Técnica em Análises Clínicas. Estudante do curso de Biomedicina no UniCEUB. E-mail: loianealves@pop.com.br.

${ }^{6}$ Mestre em Parasitologia. Orientadora do Estágio de Parasitologia. Docente do curso de Biomedicina do UniCEUB. E-mail: alveslm@gmail.com. 


\section{Introdução}

Os animais domésticos representam um importante papel na sociedade, pois eles contribuem para o bem estar do homem (ALVES; GOMES; SILVA, 2005). Todavia são hospedeiros em potencial de alguns parasitos zoonóticos e por estarem sempre presentes em locais de lazer como praças públicas e praias, o risco de infecção tem aumentado, especialmente para crianças. Essa situação gera um problema de saúde pública (CAMPOS FILHO et al., 2008), pois esses lugares constituem ambientes de transmissão de agentes patogênicos, inclusive parasitários (CORREA et al., 1995; SANTAREM; SARTOR; BERGAMO, 1998; ARAUJO et al., 1999; MERCADO et al., 2004; CAMPOS-FILHO et al., 2008).

Entre os parasitos que afetam os cães e que são capazes de causar infecções em humanos destacam-se os dos gêneros Toxocara, Ancylostoma, Cryptosporidium, Giardia e Dipilydium. Alguns podem ser agentes da larva migrans visceral, larva migrans cutânea e giardíase em humanos e animais, ocasionando distúrbios intestinais (OLIVEIRA-SEQUEIRA et al., 2002).

O estudo de busca parasitológica em fezes de cães é fundamental, pois, segundo Scaini et al. (2003), os cães desempenham um importante papel como fonte de contaminação ambiental de parasitos com potencial zoonótico, liberando cerca de 15.000 ovos por grama de fezes que podem ser encontrados em locais, principalmente de recreação, além de despertar o interesse para buscar meios profiláticos, visando à diminuição do risco de infecção para o homem e para os animais (SANTAREM; SARTOR; BERGAMO, 1998).

Segundo Alves, Gomes e Silva (2005), o conhecimento da frequência desses enteroparasitos é importante, tanto pelo caráter zoonótico como pelos danos diretos que eles causam em seus hospedeiros. O estudo desses parasitos é necessário para que medidas profiláticas de controle possam ser adotadas evitando a possibilidade de transmissão para crianças, idosos e pessoas imunocomprometidas, visando a uma boa qualidade de vida para humanos e cães.

O presente estudo teve como objetivo verificar a presença de parasitos em amostras de fezes de cães domésticos e em amostras de fezes encontradas em áreas públicas e de áreas residenciais do Distrito Federal, por meio da utilização de técnicas de diagnóstico parasitológico. 


\section{Material e métodos}

Em abril e maio de 2009, foram colhidas 20 amostras de fezes de cães selecionadas por conveniência. Destas, 7 foram provenientes de cães criados em domicílio e 13 de fezes de cães encontradas em locais públicos do Distrito Federal. A raça, o sexo e a idade dos animais foram especificados. Não foram colhidas informações concernentes ao uso de vermicida nos cães de domicílio.

As amostras de rua foram colhidas do solo durante o período da tarde, a partir das 14 horas, em uma entrequadra da Asa Norte, Brasília Distrito Federal. As amostras foram acondicionadas em frascos plásticos próprios devidamente identificados e conservadas, refrigeradas em caixa de isopor até o processamento e análises no laboratório, no mesmo dia. Contudo, as amostras que não foram analisadas no mesmo dia foram acondicionadas em formalina a 10\% para a preservação das formas parasitárias e refrigeradas em geladeira, a aproximadamente $4^{\circ} \mathrm{C}$, até o processamento e análise.

As amostras de cães domiciliados foram colhidas do solo no dia anterior ao das análises e colocadas em frascos plásticos próprios, contendo formalina a $10 \%$. Os frascos foram identificados e conservados refrigerados até serem encaminhados ao laboratório. O processamento e as análises de todas as amostras foram realizados no Laboratório de Parasitologia do Centro Universitário de Brasília UniCEUB até 48 horas após a coleta

O diagnóstico foi realizado por meio de exame direto, da técnica de flutuação em solução saturada de açúcar (solução de Sheather’s) (LEVINE, 1978), da técnica de flutuação em sulfato de Zinco (FAUST, 1939), para a deteç̧ão de ovos de helmintos, cistos e oocistos de protozoários e da técnica de sedimentação espontânea (HOFFMAN et al. 1934).

Os resultados foram analisados por meio da frequência relativa.

\section{Resultados}

Das 20 amostras coletadas, 7 (35\%) apresentaram parasitos de importância clínica para humanos e 13 (65\%) foram negativas (Tabela 1). 
O gênero Giardia sp. foi encontrado na forma de cisto em $85,7 \%$ das amostras positivas. Destas, $28,6 \%$ apresentaram poliparasitismos com frequência de 14,3\% para Giardia sp. e ancilostomídeo e 14,3\% para Giardia sp. e Toxocara sp das amostras positivas (Tabela 2). Vale ressaltar que os casos de poliparasitismo somente foram encontrados em amostras de cães de rua.

Também, pôde-se observar a presença de ovo de ancilostomídeo em 14,3\% das amostras de rua (Tabela 2).

Tabela 1 - Presença de parasitos em amostras de fezes de cães.

\begin{tabular}{|c|c|c|c|c|c|c|}
\hline \multirow{2}{*}{ Origens das amostras } & \multicolumn{7}{|c|}{ Parasitos } \\
\cline { 2 - 7 } & Positivo & $\%$ & Negativo & $\%$ & Total & $\%$ \\
\hline Cães domésticos & 1 & 5 & 6 & 30 & 7 & 35 \\
\hline Cães de rua & 6 & 30 & 7 & 35 & 13 & 65 \\
\hline Total & 7 & 35 & 13 & 65 & 20 & 100 \\
\hline
\end{tabular}

Tabela 2 - Positividade de parasitos com potencial patogênico em humanos em fezes de cães de rua do Distrito Federal.

\begin{tabular}{|l|c|c|}
\hline Parasitos & $\begin{array}{c}\text { Cães de domicílio } \\
(\%)\end{array}$ & $\begin{array}{c}\text { Cães de Rua } \\
\text { (\%) }\end{array}$ \\
\hline Ancilostomídeo & 0 & 14,3 \\
\hline Giardia $s p$. & 14,3 & 42,8 \\
\hline Giardia $s p .+$ ancilostomídeo & 0 & 14,3 \\
\hline Giardia $s p .+$ Toxocara $s p$ & 0 & 14,3 \\
\hline
\end{tabular}

\section{Discussão}

Neste estudo foram encontrados, especialmente em cães de rua, ovos de helmintos e cistos de protozoários de parasitos de relevância clínica como os do gênero Toxocara sp., Giardia sp., ancilostomídeo (NEVES, 2003; REY, 2008). Tais parasitos em contato com o organismo humano podem causar vários sintomas, que vão desde uma simples diarreia doenças graves como o amarelão (NEVES, 2003). 
Durante a pesquisa foi encontrado ovo de ancilostomídeo em 14,3\% das amostras e corroborando com achados de Blazius et al. (2005), Campos-Filho et al. (2008), Scaini et al. (2003) e Capuano e Rocha (2006), que em outros estados encontraram esse parasito na frequência variando entre 41,7\% e 71,3\%. Esses estudos tiveram como escopo analisar a contaminação de áreas públicas por fezes de cães (CAMPOS-FILHO et al., 2008). A presença de ancilostomídeos em fezes de cães não é surpreendente, já que esses animais não desenvolvem imunidade contra antígenos (BLAZIUS et al., 2005). Essa baixa frequência observada no presente estudo, em relação aos trabalhos relatados pode estar relacionada ao uso de antihelmíticos e a outros métodos de controle (ALVES et al., 2005), já que, segundo Bugg et al. (1999 apud ALVES et al., 2005, p 130) essas táticas vêm sendo estimuladas de forma mais intensa nos últimos anos. Além disso, essa relação também pode estar ocorrerendo em função da condição socioeconômica da região, já que o local selecionado para a realização da coleta de amostras de rua é uma área caracterizada por ser local de nível socioeconômico relativamente alto.

Neste estudo foram encontrados cistos de Giardia sp. em 85,7\% das amostras positivas isoladamente ou em associação. Destacando-se que, das amostras positivas para Giardia sp., 28,6 \% do total de amostras positivas apresentaram poliparasitismo na amostra com frequência de 14,3\% para Giardia sp. e ancilostomideo; e 14,3\% para Giardia sp. e Toxocara sp., contrastando com os resultados de Oliveira-Sequeira et al. (2002), Sogayar e Corrêa (1984) e Alves et al. (2005) que observaram a presença de Giardia sp. em 12,2\%, 3,4\% e 1,6\% das amostras positivas, respectivamente. A alta frequência desse protozoário encontrada neste estudo pode ser pelo fato de que a Giardia sp. tem a característica de apresentar períodos positivos e períodos negativos nas fezes (ALVES et al., 2005). Desse modo, acredita-se que tais amostras foram colhidas durante o período positivo.

Segundo Bugg et al. (1999 apud KATAGIRI, OLIVEIRA-SEQUEIRA, 2007), em países desenvolvidos, G. duodenalis tem sido o parasita entérico mais comum de cães domésticos, contudo a prevalência de Giardia é frequentemente subestimada devido à baixa sensibilidade dos métodos convencionais de diagnóstico, em virtude dos parasitas ocorrerem em níveis subclínicos e da eliminação de cistos ser naturalmente intermitente (McGLADE et al., 2003 apud KATAGIRI; OLIVEIRA-SEQUEIRA, 2007). 
Encontrou-se, também, ovo de Toxocara sp. em 14,3\% das amostras. Esse resultado se enquadra com os achados de Campos-Filho et al. (2008), Scaini et al. (2003) e Capuano e Rocha (2006) que também encontraram parasitos desse gênero variando a frequência entre 4,2 e 32,5\%. O helminto Toxocara canis é frequentemente encontrado no intestino delgado de cães (KATAGIRI; OLIVEIRA-SEQUEIRA, 2007), e é o maior responsável pela síndrome da Larva migrans visceral (ALVES et al. 2005) e algumas vezes pode afetar o globo ocular, gerando a síndrome Larva migrans ocular (SANTAREM; SARTOR; BERGAMO, 1998).

Nesta pesquisa, esse parasito foi encontrado somente em amostras de rua, e corrobora com os resultados de Alves et al. (2005) que observaram maior contaminação em amostras procedentes de cães vadios, e inferiu que cães de rua estão mais expostos a ambientes contaminados e por sua vez sujeitos ao abandono, à deficiência nutricional e a condições de maior estresse, o que os torna mais suscetíveis a infecções parasitárias. No Brasil, tem sido comum a notificação de ovos de T. canis em amostras de fezes ou solo (SCAINI et al., 2003; CAPUANO; ROCHA, 2006; CAMPOS-FILHO et al., 2008).

\section{Conclusão}

Algumas amostras de fezes de cães encontradas na quadra residencial da Asa Norte apresentaram helmintos e protozoários de importância clínica, em especial o protozoário Giardia sp., colocando em risco a saúde das pessoas. Dessa forma, medidas de controle dos animais soltos devem ser intensificadas para diminuição de exposição humana a fatores zoonóticos, garantindo uma melhor qualidade de vida à população.

Portanto, este trabalho contribui para o esclarecimento da comunidade local sobre a presença de parasitos em áreas públicas, bem como alerta sobre os riscos de infecção parasitária por fezes de cães contaminadas, pois não houve estudos precedentes sobre parasitos de importância zoonótica no Distrito Federal em animais domésticos. 


\title{
Occurence of zoonotic parasites in dog feces from a place in Asa Norte, Brasília, DF
}

\begin{abstract}
The knowledge of the frequency of parasites in dogs is important due to the zoonotic character and the damage caused to hosts. Twenty samples of feces were analyzed, 7 were collected at home and 13 were found in public places of Asa Norte - DF. Eight had eggs and cysts of parasites such as the clinical relevance of the genus Toxocara sp., Giardia sp. and eggs from the family Ancylostomatidae. This study was important to clarify the local population about the risk of contamination by the feces of dogs. The data from this study, suggest that control measures should be intensified to ensure better quality of life to the population.
\end{abstract}

Keywords: Feces of dogs, Parasitological diagnostic techniques, Frequency of parasites.

\section{Referências}

ALVES, F. O.; GOMES, A. G.; SILVA, A. C. Ocorrência de enteroparasitos em cães do município de Goiânia, Goiás: comparação de técnicas de diagnóstico. Revista Ciência Animal Brasileira, Goiás, v. 6, n. 2, p. 127-133, abr./jun. 2005.

ARAUJO F. R. et al. Contamination of public squares of Campo Grande, Mato Grosso do Sul, Brazil, with eggs of Toxocara and Ancylostoma in dog feces. Revista da Sociedade Brasileira de Medicina Tropical, Uberaba, v. 32, n. 5, p. 581-583, 1999.

BLAZIUS, R. D. et al. Ocorrência de protozoários e helmintos em amostras de fezes de cães errantes da Cidade de Itapema, Santa Catarina. Revista da Sociedade Brasileira de Medicina Tropical, Uberaba, v. 38, n. 1, p. 73-74, jan./fev. 2005.

CAMPOS FILHO, P. C. et al. Parasitas zoonóticos em fezes de cães em praças públicas do município de Itabuna, Bahia, Brasil. Revista Brasileira de Parasitologia Veterinária, São Carlos, v. 17, n. 4, p.206-209, 2008.

CAPUANO, D. M.; ROCHA, G. M. de. Ocorrência de parasitas com potencial zoonótico em fezes de cães coletadas em áreas públicas do município de Ribeirão Preto, SP, Brasil. Revista Brasileira de Epidemiologia, São Paulo, v. 9, n. 1, p. 8186, 2006. 
FAUST, E. C et al. Comparative efficiency of various technics fo the diagnosis of protozoa and helminthes in feces. Journal of Parasitology, New York, v. 25, p. 241-62, 1939.

HOFFMAN, W. A. etal. The sedimentation concentration method in schistosomiasis mansoni. Puerto Rico Journal of Public Health and Tropical Medicine, Puerto Rico, v. 9, p. 283-298, 1934.

KATAGIRI, S.; OLIVEIRA-SEQUEIRA, T. C. G. Zoonoses causadas por parasitas intestinais de cães e o problema do diagnóstico. Arquivo do Instituto de Biologia, São Paulo, v. 74, n. 2, p. 175-184, abr./jun., 2007.

LEVINE, N.D. Textbook of veterinary: parasitology. Minneapolis: Burges, 1978.

MERCADO, R. et al. Exposure to larva migrans syndromes in squares and public parks of cities in Chile. Revista de Saúde Pública, São Paulo, v. 38, n. 5, p. 729-731, 2004.

NEVES, D. P. Parasitologia humana. 10. ed. São Paulo: Atheneu, 2003.

OLIVEIRA-SEQUEIRA, T. C. G. et al. Prevalence of intestinal parasites in dogs from São Paulo State, Brazil. Veterinary Parasitology, USA, v. 103, n. 1-2, p. 1927, 2002.

SANTAREM, V. A.; SARTOR, I. F.; BERGAMO, F. M. M. Contaminação, por ovos de Toxocara spp, de parques e praças públicas de Botucatu, São Paulo, Brasil. Revista da Sociedade Brasileira de Medicina Tropical, Uberaba, v. 31, n. 6 , p. 529-532, 1998.

SCAINI C. J. et al. Environmental contamination by helminth eggs and larvae in dog feces from central area of Cassino beach, Rio Grande do Sul. Revista da Sociedade Brasileira de Medicina Tropical, Uberaba, v. 36, n. 5, p. 617-619, 2003.

SILVA, T.P. et al. Dinâmica da infecção natural por Eimeria spp. em cordeiros da raça Santa Inês criados em sistema semi-intensivo no Norte de Minas Gerais. Arquivo Brasileiro de Medicina Veterinária de Zootecnia, Belo Horizonte, v. 59, n. 6, p. 1468-1472, 2007.

SOGAYAR, M. I. L.; CORRÊA, F. M. A. Giardia in dogs in Botucatu, São Paulo state, Brazil: a comparative study of canine and human species. Revista de Ciências Biomédicas, São Paulo, v. 5, n. 6, p. 69-73, 1984. 
REY, L. Parasitologia: parasitos e doenças parasitárias do homem nos trópicos ocidentais. 4. ed. Rio de Janeiro: Guanabara Koogan, 2008.

TAMEKUNI, K. et al. Eimeriose em bovinos de corte da região de Londrina, Paraná. In: ENCONTRO ANUAL DE INICIAL CIENTÍFICA, 11., 2002, Maringá. Anais.... Maringá: EDUEM, 2002. v. 11. 
Para publicar na revista Universitas Ciências da Saúde, entre no endereço eletrônico www.publicacoesacademicas.uniceub.br. Observe as normas de publicação, facilitando e agilizando o trabalho de edição. 